\title{
Factores edáficos-fisiográficos
}

\author{
y calidad de sitio del Pinus \\ occidentalis Sw.
}

\section{Edaphic-physiographic factors and site quality of Pinus occidentalis Sw.}

\author{
Santiago W. Bueno-López ${ }^{1 *}$ Juan Gilberto Torres Herrera' y Milton García'
}

\begin{abstract}
Programa de Investigaciones Silviculturales, Ambientales y Cambio Climático. Vicerrectoría de Investigación e Innovación. Pontificia Universidad Católica Madre y Maestra. Santiago. República Dominicana. mgarcia@gmail.com.
\end{abstract}

\author{
* Autor para correspondencia: sbueno@pucmm.edu.do, \\ swbueno@gmail.com.
}

\section{RESUMEN}

La estimación de la calidad de sitios para la producción forestal normalmente se logra aprovechando la presencia de árboles que exhiben diferentes patrones de crecimiento en altura y desarrollando ecuaciones que relacionan su altura y edad. Sin embargo, hay lugares desprovistos de árboles donde la inversión en bosques productivos es factible; más aún si se puede estimar a priori la calidad potencial de regenerar determinadas especies forestales. Se desarrollaron modelos matemáticos regresivos para estimar el índice de sitio en rodales naturales de Pinus occidentalis Sw., en las principales regiones productivas de Republica Dominicana, estableciendo también la relación entre el índice de sitio y variables edáficas y fisiográficas. Los modelos permiten evaluar el potencial productivo en lugares desprovistos de árboles. No se encontraron diferencias significativas $(\alpha 0.01)$ entre los índices de sitio promediados por parcela en cada localidad, pero sí se obtuvo una correlación significativa entre el índice de sitio, altura sobre el nivel del mar y exposición de la respectivamente. Una correlación significativa fue también encontrada entre índice de sitio y contenido de materia orgánica, sodio y potasio . Las variables elevación sobre el nivel del mar y azimut resultaron estadísticamente significativas y pueden utilizarse para predecir el potencial productivo de lugares donde no existen árboles. La estimación del potencial productivo mediante variables edáficas y fisiográficas para los rodales naturales de $P$. occidentalis $\mathrm{Sw}$. en las regiones productivas, constituye una herramienta indispensable en el manejo sostenible de estas áreas boscosas.

PALABRAS ClAVE: curvas guía anamórficas, modelos matemáticos regresivos, Pinus occidentalis Sw., gestión sostenible, productividad potencial.

\begin{abstract}
Site quality estimation for forest production is normally accomplished by estimating height growth potential of forest stands, by developing equations that relate height and age of sample trees. However, there are places devoid of trees where investment in productive forests is feasible; better yet if quality potential of regenerating certain forest species can be estimated a priori. We have developed regressive mathematical models to estimate site index in natural stands of Pinus occidentalis, in major productive regions in the Dominican Republic, and established the relationship between index site and edaphic and physiographical variables. Developed models allow the assessment of the productive potential in places without trees. There were no significant differences $(\alpha=0.05)$ between the site indexes averaged per plot in each locality, but a significant correlation was obtained among site index, height above sea level and aspect $(0.273$, $\mathrm{P}=0.000$ and $0.207, \mathrm{P}=0.003)$ respectively. There were also significant correlations between site index and soil organic matter content within the soil, sodium and potassium. The variables "height above sea level" and azimuth were statistically significant, and can be used to predict the productive potential of places where there are no trees within this region. The estimate of the productive potential by edaphic and physiographical variables for natural stands of $P$. occidentalis in productive areas constitutes an essential tool in the sustainable management of these forested areas.
\end{abstract}

KEYwORDS: anamorphic guide curves, regressive mathematical models, Pinus occidentalis Sw., sustainable management, potential forest productivity. 


\section{INTRODUCCIÓN}

El pino criollo o pino de cuaba Pinus occidentalis Sw. es endémico de La Española. Se encuentran en poblaciones mixtas con árboles de hoja ancha, por debajo de los 2100 $\mathrm{m}$ snm y en rodales puros por encima de estas elevaciones (Farjon et al., 1997). Este tipo de bosque se desarrolla adecuadamente por encima de los $800 \mathrm{~m}$ snm y son la única formación boscosa por encima de los $2100 \mathrm{~m}$. En tierras bajas, el pino criollo se desarrolla particularmente en suelos lateríticos y climas muy húmedos. Tiene la capacidad de crecer en suelos infértiles, ácidos, superficiales debido a que son capaces de establecer relaciones simbióticas con ectomicorrizas.

En República Dominicana, el bosque de coníferas constituido por rodales puros y mixtos de la especie, en la actualidad abarca un área de $1300 \mathrm{~km}^{2}$, de la cual e $69.2 \%$ es zona de producción. Existen 462 productores de bosque conífero establecidos en 38785 ha (43\%) bajo regencia de planes de manejo. De acuerdo con FAOSTAT (base de datos estadísticos de la Organización de las Naciones Unidas para la Alimentación y la Agricultura), en el año 2012 se registró una producción de $10000 \mathrm{~m}^{3}$ de madera aserrada de pino y se importaron $180000 \mathrm{~m}^{3}$. No obstante la importancia de la producción maderera, se desconoce a nivel local la capacidad productiva de los sitios forestales (Rodríguez, 2008).

El índice del sitio (IS) indica la altura media de árboles dominantes a una edad determinada y se utiliza a menudo para estimar la productividad potencial de los sitios boscosos, constituyendo una herramienta esencial de soporte para el manejo apropiado de los bosques (Mathiasen et al., 2006).

El método más común de estimar índice de sitio en la actualidad es el uso de curvas de índice de sitio, que muestran la relación entre la altura y edad (Avery y Burkhart, 2002; Husch et al., 2003, West, 2003). El rodal bajo estudio se utiliza como una prueba biológica de la productividad del sitio. La metodología es válida solo si la condición del rodal es tal que el índice de sitio estimado no está indebidamente influenciado por el manejo silvicultural a que ha sido sometido el bosque. En otras palabras, el rodal debe estar intacto, ser de una sola especie, coetáneo y lo más homogéneo en sus características fenotípicas y genotípicas.

Los países con alta tradición forestal han desarrollado por varias décadas modelos para índice de sitio de sus principales especies maderables. Los investigadores han utilizado diversas técnicas estadísticas y diferentes variables explicadoras para predecir la altura total y el índice de sitio. Dobler (1999) ha desarrollado una serie de curvas de índice de sitio para P. occidentalis Sw. en el área de influencia del Plan Sierra y es el único registro encontrado en la literatura de trabajos de esta índole para el país. Carmean (1972) es de los primeros en desarrollar curvas de índice de sitio para robles en los estados centrales de Estados Unidos. Utilizando los datos de análisis del fuste, este autor encontró que las cuatro especies de roble estudiadas poseen diferente patrón de crecimiento en altura y esos patrones resultaron polimórficos. Curtis et al. (1973) estudiaron las diferencias que resultan de utilizar Índice de Sitio y Altura como variables dependientes, intercambiando cada una de estas variables de dependiente a independiente además de la edad.

Chile, Argentina y México han desarrollado modelos de índice de sitio para sus especies principales. RodríguezAcosta y Arteaga-Martínez (2005) estimaron ecuaciones de índice de sitio para Pinus chiapensis en los estados de Veracruz y Puebla, México. Essea et al. (2007) trabajaron la construcción de curvas de índice de sitio para renovales de coigüe (Nothofagus dombeyi) en la precordillera andina de la IX Región, Chile, a partir del análisis espacial de las variables fisiográficas altitud, exposición y pendiente. Andenmatten y Letourneau (2000) establecieron curvas de índice de sitio y crecimiento en altura para pino Oregón (Pseudotsuga menziesii), para ser aplicadas en la región andino patagónica de Argentina, mediante el análisis fustal de 69 árboles dominantes, seleccionados de 28 rodales. López-Hernández (2010), probó los modelos de crecimiento Logístico Gompertz y Schumacher para generar curvas anamórficas de índice de sitio para Pinus patula, a partir de datos edad-altura dominante obtenidos de análisis troncales en la zona de aprovechamiento fores- 
tal maderable intensivo de la comunidad de Santiago Comaltepec, Ixtlán, Oaxaca.

Monserud (1984) obtuvo datos para el abeto Douglas en rodales coetáneos y disetáneos de las Montañas Rocosas, utilizando la técnica de regresión logística generalizada. Además, encontró que utilizar el tipo de hábitat como variable concomitante fue útil para mejorar la predicción del modelo. Los datos fueron analizados incluyendo un componente de varianza auto-regresivo de primer orden, dada la naturaleza de las mediciones (repetidas en los mismos sujetos), lo que podría provocar auto correlación alta en los parámetros estimados. Zhang et al. (1993) estudiaron los atributos asintóticos de rodales forestales como la altura total basándose en un raciocinio bio-matemático. En Europa, Nord-Larsen (2006) desarrolló curvas de índice de sitio para el haya europea (Fagus sylvatica L.) y Elfving y Kiviste (2007), de la Universidad Sueca de Ciencias Agrícolas y de la Universidad Agrícola de Estonia, respectivamente, construyeron ecuaciones de índice de sitio para Pinus sylvestris L. utilizando datos provenientes de parcelas permanentes en Suecia. Estos autores probaron los tres métodos comúnmente utilizados para desarrollar ecuaciones de índice de sitio, la curva guía, la predicción de parámetros y la ecuación diferencial. Otra técnica estadística utilizada es la Línea de Regresión Media Geométrica (Nigh, 1995).

\section{OBJETIVOS}

El objetivo principal del estudio fue desarrollar las ecuaciones de índice de sitio con el fin de evaluar la calidad para la producción de Pinus occidentalis en tres zonas productoras de madera de República Dominicana: 1) Jarabacoa, 2) San José de Las Matas y 3) Restauración. Además, analizar los principales factores edáficos $\mathrm{y}$ fisiográficos que afectan la calidad de los sitios intervenidos y relacionarlos mediante modelos de regresión al índice de sitio correspondiente.

\section{MATERIALES Y MÉTODOS}

El estudio incluyó tres zonas de bosques de P. occidentalis Sw. en la Cordillera Central: 1) Jarabacoa, 2) San José de
Las Matas y 3) Restauración. La zona de Jarabacoa tiene como coordenadas centrales UTM 19Q 242515mE y $2133996 \mathrm{mN}$, a una altura media de $529 \mathrm{~m} \mathrm{snm}$. Temperatura media anual inferior a $18{ }^{\circ} \mathrm{C}$ y precipitación en el intervalo de $1000 \mathrm{~mm} / \mathrm{año}$ a $1500 \mathrm{~mm} /$ año. Las zonas de vida predominantes (Holdridge, 1987) son el bosque muy húmedo montano bajo (Mmh-MB) y el bosque muy húmedo montano (Mmh-M). En esta zona se establecieron 14 parcelas en bosque húmedo y 8 en bosque de transición de seco a húmedo.

La zona de San José de Las Matas tiene las coordenadas centrales UTM 19Q $295125 \mathrm{mE}$ y $2138513 \mathrm{mN}$, con un con altitud entre $400 \mathrm{~m}$ snm y más de $1000 \mathrm{~m}$ snm y una media de $750 \mathrm{~m}$ snm. La temperatura media anual es de $24{ }^{\circ} \mathrm{C}$, con variaciones entre máxima y mínima de menos de $10^{\circ} \mathrm{C}$. Las zonas de vida predominantes son: Bosque Subtropical Seco (BSs), Bosque Muy Húmedo Subtropical (BMHS) y, Bosque Húmedo Subtropical (BHS). En esta zona se establecieron 14 parcelas en bosque húmedo y 8 en bosque de transición de seco a húmedo (Holdridge, 1987).

La zona de Restauración tiene las coordenadas UTM centrales 19Q $216880 \mathrm{mE}$ y $2137602 \mathrm{mN}$, con una altitud media de $594 \mathrm{~m} \mathrm{snm}$. La temperatura media anual es de $18{ }^{\circ} \mathrm{C}$, con una precipitación media anual de $1600 \mathrm{~mm} /$ año. Las zonas de vida predominantes son: Bosques Húmedo Subtropical (Bh-S), Bosque Húmedo Montano (Bh-M) y Bosque muy Húmedo Subtropical (Bmh-S). Aquí se establecieron 14 parcelas en bosque húmedo y 8 en bosque de transición de seco a húmedo (Holdridge, 1987).

\section{Selección de Parcela}

Las parcelas fueron seleccionadas combinando varias características, de manera que la probabilidad de un manejo indeseado del rodal sea razonablemente baja. En ese sentido, se tomaron en cuenta los criterios siguientes:

1. La edad media a la altura del pecho de los árboles de altura dominante y co-dominante en una parcela entre 20 años y 90 años; 
2. El rodal debía ser coetáneo con una diferencia de edad entre árboles más jóvenes y más viejos que no excediera 20 años (Nyland, 2002);

3. La densidad del rodal seleccionado fue de por lo menos $40 \%$ de un rodal con densidad relativa máxima;

4. Los árboles con altura dominante y co-dominante no podían presentar los daños siguientes: rotura, mortalidad en la parte superior, madera doblada, daños por los ataques de insectos y

5. Al menos 70\% del área basal en la parcela debía ser $P$. occidentalis Sw.

\section{Levantamiento de Datos}

Los datos de altura total se obtuvieron seleccionando de tres a cinco árboles, los de mayor diámetro, en las clases dominantes y co-dominante dentro de cada parcela. La edad se determinó mediante conteo de anillos en viruta extraída con barreno (Burgt, 1993) y el DAP (diámetro a la altura del pecho) se midió a $1.30 \mathrm{~m}$ sobre el suelo.

Se tomaron muestras de suelo de cada parcela, siguiendo los procedimientos estándares, para análisis en laboratorio de las propiedades químicas y físicas. Los datos geográficos incluyeron elevación sobre el nivel del mar, latitud y longitud en coordenadas UTM y exposición de la pendiente. Las variables topográficas incluyeron: pendiente, posición de la parcela en la pendiente, forma del terreno, forma del relieve y complejidad de la pendiente.

\section{Estimación de la Edad}

La edad del rodal se determinó por conteo de anillos utilizando los protocolos desarrollados por Burgt (1993) y Dobler (1999). En los trópicos, el crecimiento de los árboles no se detiene como ocurre en las zonas templadas durante el invierno. Es influenciado por las temporadas de lluvia, limitándose seriamente su crecimiento en períodos secos. En la región de estudio hay una importante temporada de lluvia al año, que ocurre en los meses de abril y mayo y dos períodos de menor cantidad de precipitación en septiembre-octubre y diciembre. Se utilizó la metodolo- gía de Dobler (1999) para las localidades de Jarabacoa y Restauración. Para San José de Las Matas se asumieron los hallazgos de ambos autores para el conteo de anillos por año. Dobler (1999) encontró que se forman 2.86 anillos cada año en las zonas intermedia y húmeda de San José de las Matas. Para la zona de Jarabacoa, se encontró que se producen 2.53 anillos por año en promedio y, para la zona de Restauración 1.39 anillos por año.

\section{Modelos Matemáticos}

El modelo general de evaluación del índice se integró con tres categorías de variables, expresado de la forma siguiente:

Índice de Sitio = f(var. edáficas, var. topográficas, var.climáticas)

El índice de sitio (S) se obtuvo empleando el modelo de Schumacher (1939) como lo describe Clutter et al. (1983), mediante la función:

$$
\operatorname{In}(S)=\ln (H)-\hat{\beta}_{1}\left(E_{1}^{-1}-E_{0}^{-1}\right)
$$

Donde:

$\mathrm{S} \quad=$ índice de sitio

$\mathrm{H} \quad=$ Altura $(\mathrm{m})$

$\mathrm{E}_{1} \quad=$ edad actual $\mathrm{o}$ asumida (años)

$\mathrm{E}_{0} \quad=$ edad índice o base (40 años)

$\beta_{1} \quad=$ coeficiente de pendiente a ser estimado

El coeficiente beta sub cero $\left(\beta_{0}\right)$ de la ecuación de Schumacher se determinó aplicando el modelo regresivo que relaciona la altura y la edad mediante la ecuación siguiente:

$$
\operatorname{In}(\mathrm{H})=\hat{\beta} 0+\hat{\beta} 1\left({ }^{1} / \mathrm{E}\right)
$$

Donde:

$\mathrm{E}=$ Edad actual (años)

$\mathrm{H}=$ Altura promedio $(\mathrm{m})$ de árboles en cada parcela

$\beta_{0} \mathrm{y} \beta_{1}=$ coeficientes a ser estimados 
Se adoptó una edad índice de 40 años, la cual se aproxima a la edad de rotación de la especie bajo estudio. De la curva guía ajustada mediante la ecuación [1] se derivó el conjunto de curvas de índice de sitio anamórficas, para índices de sitio de 20, 25, 30, 35 y 40; en cada municipio. El coeficiente de cada índice de sitio fue recalculado mediante la ecuación:

$$
\hat{\beta}_{0}=\operatorname{In}(S)-\hat{\beta}_{1} * 1 / E_{0}
$$

Con los $\beta_{0}$ re-calculados se construyeron las curvas anamórficas (Goelz y Burk, 1992), retransformando desde la escala logarítmica mediante la siguiente ecuación:

$$
\mathrm{S}=\operatorname{Exp}\left(\hat{\beta}_{0}\right) * \operatorname{Exp}\left(\hat{\beta}_{1} / \mathrm{E}_{0}\right)
$$

A partir de la ecuación (4) se generaron los datos para la construcción de las curvas anamórficas para cada índice de sitio. A su vez, los índices de sitio retransformados a partir de la ecuación [1], se utilizaron como variable dependiente de los modelos de regresión múltiple, para identificar las variables ambientales estadísticamente significativas y predecir la capacidad productiva potencial de los rodales en las regiones estudiadas. Las características topográficas y las propiedades físico-químicas del suelo se utilizaron como las variables predictoras. El análisis de los datos y el procedimiento para el desarrollo de los modelos fueron ejecutados usando SAS (Statistical Analysis System Inc., 1990).

Se hizo un análisis de regresión de un modelo completo, utilizando todas las variables de predicción numérica, suponiendo que la relación entre índice de sitio (IS) y cada variable predictiva es lineal. El modelo general empleado es de la forma:

$$
Y_{i}=\beta_{0}+\beta_{1} X_{1}+\beta_{2} X_{2}+\ldots+\beta_{m} X_{m}+\varepsilon_{i}
$$

Donde:

$$
\begin{aligned}
\mathrm{Y}_{\mathrm{i}} & =\text { Índice de Sitio estimado } \\
\mathrm{X}_{1}, \mathrm{X}_{2}, \ldots, \mathrm{X}_{\mathrm{m}}= & \text { variables topográficas, edáficas y cli- } \\
& \text { máticas }
\end{aligned}
$$

$\beta_{0}, \beta_{1}, \beta_{2}, \ldots, \beta_{\mathrm{m}}=$ Coeficientes de regresión a ser estimados

$\varepsilon_{\mathrm{i}}=$ Término de error

Los modelos competidores se compararon mediante regresión múltiple y se seleccionó el "mejor" siguiendo la técnica de regresión paso a paso (Stepwise regression), considerando los criterios siguientes: i) Significación estadística de cada variable en el modelo $(\alpha=0.05)$ y ii) Coeficiente de determinación del modelo $\left(\mathrm{R}^{2}\right)$. Para la sección de variables estadísticamente significativas se utilizó la prueba $\mathrm{F}$ con niveles alfa de 0.01 y 0.05 , para los niveles de entrada y remoción de variables, respectivamente.

\section{RESULTADOS}

El modelo [3] ha sido ajustado a los datos mediante análisis de regresión para cada árbol en la muestra, lográndose obtener como resultado el índice de sitio promediado por parcela. Un análisis de varianza indico que, a un nivel alfa de 0.01 (99\% de confianza), no hay diferencias significativas entre los índices de sitio promediados por parcela en cada municipio. Para los rodales en Jarabacoa, San José de las Matas y Restauración, los coeficientes e índices de sitio obtenidos por medio de las ecuaciones [2] y [3] se muestran en la tabla 1.

El gráfico de las medias (Fig. 1) indica que en promedio, el índice de sitio de los rodales en Jarabacoa es menor que el índice de sitio en las demás localidades.

Con los índices de sitio obtenidos, se construyeron las curvas anamórficas, las cuales presentan el patrón que ha de seguir el desarrollo en altura de los árboles en cada una de las localidades estudiadas a medida que avanza la edad del rodal. La figura 2 muestra en rojo las curvas correspondientes para los índices de sitio 20, 25, 30, 35 y 40 en San José de las Matas. Asimismo, las curvas azules y negras muestran los patrones del desarrollo en altura para los Índices de Sitio correspondientes en los municipios de Restauración y Jarabacoa, respectivamente. Claramente, y como se indicara en la gráfica de las medias promedio, los índices de sitio son ligeramente mayores en San José de las Matas, aunque no estadísticamente diferentes (alfa = 0.01). 
TABLA 1. Coeficientes correspondientes a cada índice de sitio y por localización, para los modelos [2] y [3].

\begin{tabular}{|c|c|c|c|c|}
\hline \multirow[b]{2}{*}{ Localización } & \multirow[b]{2}{*}{ Índice de Sitio } & \multicolumn{2}{|c|}{ Modelo [2] } & \multirow{2}{*}{$\begin{array}{c}\text { Modelo [3] } \\
\text { Recalculado }\end{array}$} \\
\hline & & $\beta_{0}$ & $\beta_{1}$ & \\
\hline \multirow{6}{*}{ Jarabacoa } & 20 & \multirow{6}{*}{3.2} & \multirow{6}{*}{-3.42} & 3.08 \\
\hline & 25 & & & 3.3 \\
\hline & 30 & & & 3.49 \\
\hline & 35 & & & 3.64 \\
\hline & 40 & & & 3.77 \\
\hline & 45 & & & 3.89 \\
\hline \multirow{6}{*}{$\begin{array}{c}\text { San José de } \\
\text { las Matas }\end{array}$} & 20 & \multirow{6}{*}{3.4} & \multirow{6}{*}{-8.96} & 3.22 \\
\hline & 25 & & & 3.44 \\
\hline & 30 & & & 3.63 \\
\hline & 35 & & & 3.78 \\
\hline & 40 & & & 3.91 \\
\hline & 45 & & & 4.03 \\
\hline \multirow{6}{*}{ Restauración } & 20 & \multirow{6}{*}{3.31} & \multirow{6}{*}{-6.14} & 3.15 \\
\hline & 25 & & & 3.37 \\
\hline & 30 & & & 3.55 \\
\hline & 35 & & & 3.71 \\
\hline & 40 & & & 3.84 \\
\hline & 45 & & & 3.96 \\
\hline
\end{tabular}

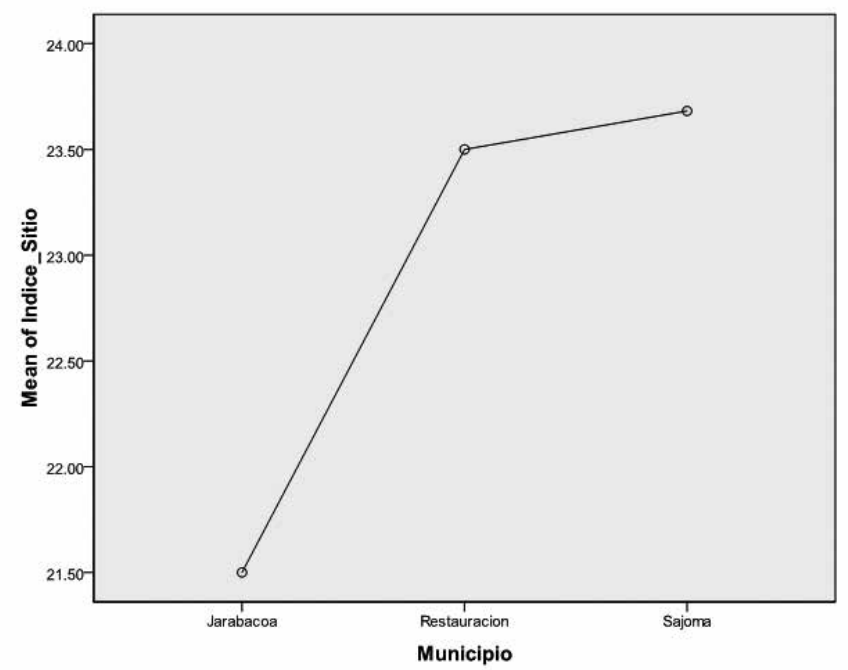

Figura 1. Gráfico de las medias promedio por parcela en cada municipio.

\section{Índice de sitio}

\section{en función de las variables topográficas}

Al no haber diferencias significativas de los índices de sitio en las tres diferentes localidades, se procedió a ajustar los datos de las variables dependientes e independientes de forma parcial, es decir, ajustando al modelo [6] las variables topográficas, las edáficas y las climáticas de manera separada. Luego, todas las variables que resultaron estadísticamente significativas (alfa $=0.05$ ) para cada tipo de propiedad (topográfica, edáfica y climática) fueron empleadas en un modelo único, con el propósito de verificar su influencia conjunta en el índice de sitio. El principio fundamental en este análisis minimiza la suma residual de cuadrados. El modelo del índice de sitio como variable dependiente con las variables topográficas, incluyó las variables explicativas elevación (ELEVAC), porciento de pendiente (PEND_PORC), exposición de la pendiente (AZIMUT_EXP), forma del terreno (FORM_TERR), tipo de pendiente (PEND_TIPO), forma del relieve (RELIEV_FORM), la posición de la parcela en la pendiente (PLOT_POS_PEND). Un gráfico P-P de probabilidad normal, sugiere que los datos provienen de una población normalmente distribuida.

Las estadísticas descriptivas de estas variables se despliegan en la tabla 2. Las últimas cuatro variables fueron introducidas en el modelo como variables indicadoras.

Hay un riesgo evidente de "sobre-ajustar" el modelo (Gardner, 1972) y, por lo tanto, crear relaciones parciales que son más o menos absurdas. Afortunadamente, la metodología paso a paso empleada en el análisis de regresión, permite extraer del modelo las variables que por motivos de colinealidad y significancia estadística no son importantes en describir la variabilidad en la variable dependiente.

El procedimiento paso a paso, con iteraciones de entrada y de remoción a un nivel alfa de 0.01 y 0.05 , respectivamente, para las pruebas $F$ excluyó todas las variables topográficas excepto la Elevación (m snm) y el Azimut. El coeficiente de determinación del modelo final fue de 0.14 , lo cual se considera muy bajo. Sin embargo, esta es la función que resulta en la menor suma de cuadrados. La 


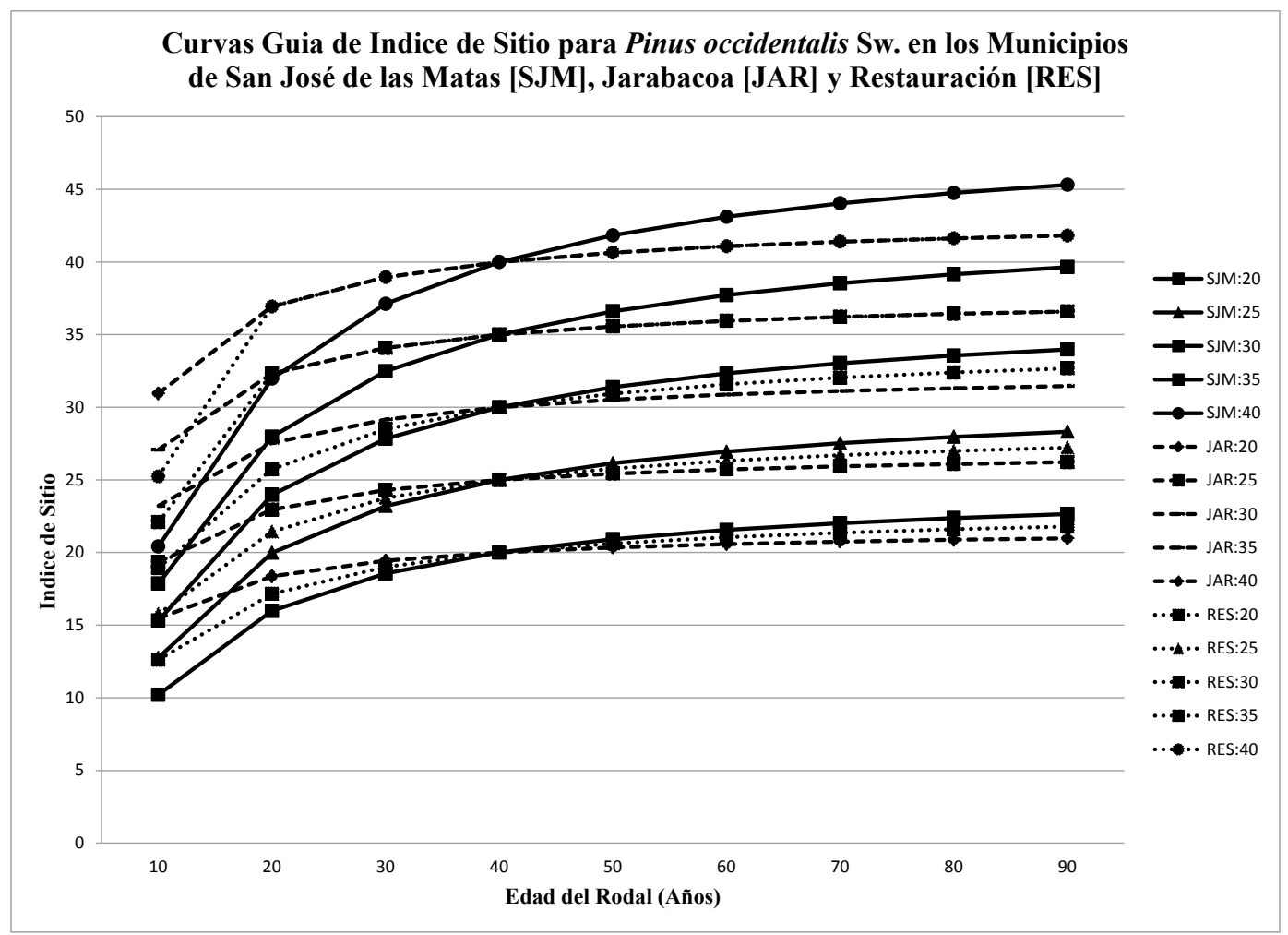

Figura 2. Conjunto de curvas anamórficas de Índice de Sitio para los rodales naturales de Pinus occidentalis Sw. en los principales municipios productores de madera en la vertiente norte de la Cordillera Central.

TABla 2. Estadística descriptiva para la variable dependiente (Índice de Sitio) y las variables topográficas.

\begin{tabular}{cccc}
\hline Variables & Promedio & $\begin{array}{c}\text { Deviación } \\
\text { Estándar }\end{array}$ & $\mathrm{N}$ \\
\hline Índice de Sitio & 22.84 & 3.39 & 178 \\
Elevación (m snm) & 709.38 & 152.48 & 178 \\
Pendiente (\%) & 31.12 & 13.35 & 178 \\
AZIMUT & 169.13 & 117.96 & 178 \\
Severidad de pendiente & 1.74 & 0.80 & 178 \\
Tipo de pendiente & 1.16 & 0.37 & 178 \\
Forma del relieve & 1.88 & 1.11 & 178 \\
Posición del centro de la parcela & 1.78 & 0.64 & 178 \\
en la pendiente & & & \\
\hline
\end{tabular}

ecuación de regresión que relaciona el índice de sitio con las dos variables significativas es la siguiente,

$$
Y_{i}=16.68+0.007 \mathrm{ELEV}+0.007 \mathrm{AZIMUT}+\varepsilon_{i}
$$

Donde:

$Y_{i} \quad=$ Índice de Sitio estimado

$X_{1}, X_{2}, \ldots, X_{m}=$ variables topográficas

$\beta_{0}, \beta_{1}, \beta_{2}, \ldots, \beta_{m}=$ Coeficientes de regresión a ser estima-

$$
\begin{aligned}
& \text { dos } \\
\varepsilon_{\mathrm{i}} & =\text { Término de error }
\end{aligned}
$$

\section{Índice de sitio en}

\section{función de las variables edáficas físicas}

Utilizando de nuevo el modelo [6], se procedió a evaluar la dependencia del Índice de Sitio con las variables edáficas físicas. Se incluyeron como variables explicativas, la profundidad del suelo (PROF) y la variable indicadora tex- 
tura del suelo (TEXT). También se incluyó de manera individual el porcentaje de arena (\%ARENA), el porcentaje de arcilla (\%ARC) y el porcentaje de limo (\%LIM). El grafico P-P de probabilidad normal para estas variables muestra desviaciones intermedias de la normalidad.

Sin embargo, al correrse el modelo de regresión paso a paso, con iteraciones de entrada y de remoción semejantes a las empleadas con las variables topográficas, un nivel alfa de 0.01 y 0.05 , solo resultó incluido en el modelo final, el porcentaje de arena (\%ARENA). El coeficiente de determinación del modelo final fue de 0.11 , indicando que es muy poca la variabilidad que se observa en índice de sitio que es explicada por el modelo. Sin embargo, como en el caso anterior, esta es la función que resulta en la menor suma de cuadrados. La ecuación de regresión que relaciona el índice de sitio con la variable significativa es la siguiente.

$$
Y_{i}=19.408+0.068 \% \text { ARENA }+\varepsilon_{\mathrm{i}}
$$

Donde:

$Y_{i} \quad=$ Índice de Sitio estimado

$X_{1}, X_{2}, \ldots, X_{m}=$ variables edáficas físicas

$\beta_{0}, \beta_{1}, \beta_{2}, \ldots, \beta_{m}=$ Coeficientes de regresión a ser estimados

$\varepsilon_{\mathrm{i}} \quad=$ Término de error

\section{Índice de sitio}

\section{en función de las variables edáficas químicas}

La dependencia del Índice de Sitio con las variables edáficas químicas se modeló utilizando variables explicativas reacción del suelo $(\mathrm{pH})$, contenido de materia orgánica (MO), fósforo asimilable (P_ASIM) en ppm, los iones intercambiables medidos en mili equivalentes por $100 \mathrm{~g}$ (Meq/100g), calcio $(\mathrm{CA})$, magnesio $(\mathrm{Mg})$, sodio $(\mathrm{Na})$, potasio $(\mathrm{K})$ y la capacidad de intercambio de cationes (CEC) también expresada en Meq/100g. El grafico P-P de probabilidad normal para estas variables muestra evidencias de auto correlación y desviaciones fuera de la normalidad.

Las estadísticas descriptivas de estas variables se despliegan en la tabla 3. Índice de sitio estuvo significativa-
Tabla 3. Estadística descriptiva para la variable dependiente (Índice de Sitio) y las variables edáficas químicas.

\begin{tabular}{cccc}
\hline Variables & Promedio & $\begin{array}{c}\text { Deviación } \\
\text { Estándar }\end{array}$ & $\mathrm{N}$ \\
\hline ÍNDICE_SITIO & 22.85 & 3.39 & 178 \\
pH & 5.74 & 0.45 & 178 \\
MO & 2.57 & 1.13 & 178 \\
Fosforo Asimilable (ppm) & 6.71 & 18.20 & 178 \\
Calcio (meq/10Og) & 4.48 & 4.34 & 178 \\
Magnesio (meq/10Og) & 3.33 & 3.22 & 178 \\
Sodio (meq/10Og) & 0.33 & 0.13 & 178 \\
Potasio (meq/100g) & 0.16 & 0.10 & 178 \\
CEC (meq/100g) & 10.14 & 9.27 & 178 \\
\hline
\end{tabular}

mente correlacionado a un nivel alfa de 0.05 y de manera positiva con el contenido de materia orgánica $(\mathrm{MO}, r=$ 0.347 , Valor_P $=0.000)$, el contenido de sodio $(\mathrm{Na}, r=$ 0.162 , Valor_P $=0.015)$, el contenido de potasio $(\mathrm{K}, r=$ 0.236 , Valor_P $=0.001)$. La correlación de la variable dependiente con la capacidad de intercambio de cationes fue negativa (CEC, $r=-0.219$, Valor_P $=0.002$ ).

$\mathrm{Al}$ correrse el modelo de regresión paso a paso, con iteraciones de entrada y de remoción semejantes a las empleadas con las variables topográficas, un nivel alfa de 0.01 y 0.05 , solo resultó incluido en el modelo final, el contenido de materia orgánica (MO). El coeficiente de determinación del modelo final fue de 0.12 , indicando que es muy poca la variabilidad que se observa en índice de sitio que es explicada por el modelo. De nuevo, como en el caso anterior, esta es la función que resulta en la menor suma de cuadrados. La ecuación de regresión que relaciona el Índice de Sitio con la variable significativa es la siguiente.

$$
Y_{i}=20.167+1.042 \mathrm{MO}+\varepsilon_{i}
$$

Donde:

$Y_{i} \quad=$ Índice de Sitio estimado 
$X_{1}, X_{2}, \ldots, X_{m}=$ variables edáficas químicas

$\beta_{0}, \beta_{1}, \beta_{2}, \ldots, \beta_{m}=$ Coeficientes de regresión a ser estimados

$\varepsilon_{\mathrm{i}} \quad=$ Término de error

\section{Índice de sitio en función}

de las variables significativas provenientes de la modelación parcial por grupos de variables

Se procedió a modelar la dependencia del índice de sitio, de las variables que en los modelos parciales [7], [8] y [9] resultaron estadísticamente significativas. Estas variables fueron la elevación sobre el nivel del mar (metros), el azimut (grados), el porciento de arena del suelo y el contenido de materia orgánica. El coeficiente de correlación en esta nueva modelación que incluye las variables significativas resultantes del ajuste parcial de variables según su tipo, muestra una mejora para las variables AZIMUT, \%ARENA y MO. El coeficiente de correlación entre IS y AZ aumentó de 0.207 a 0.219 ; el del \% ARENA desde 0.331 a 0.380 y el de MO desde 0.347 a 0.351 . Todas las correlaciones entre IS y las cuatro variables incluidas en el modelo final fueron significativas. El coeficiente de correlación más alto fue entre IS y \%ARENA $(r=0.38$, Valor_P $=0.000)$, así como entre IS y MO $(r=0.35$, Valor_P $=0.000)$.

$\mathrm{Al}$ correrse el modelo de regresión paso a paso, con iteraciones de entrada y de remoción con un nivel alfa de 0.01 y 0.05 , el modelo final resultante incluye las variables edáficas ajustadas, \%ARENA y MO. La ecuación de regresión que relaciona el índice de sitio con las variables significativas es la siguiente.

$Y_{i}=15.558+0.088 \%$ ARENA $+1.172 \mathrm{MO}+\varepsilon_{i}$

Donde:

$Y_{i} \quad=$ Índice de Sitio estimado

$X_{1}, X_{2}, \ldots, X_{m}=$ variables edáficas

$\beta_{0}, \beta_{1}, \beta_{2}, \ldots, \beta_{m}=$ Coeficientes de regresión a ser estimados

$\varepsilon_{\mathrm{i}} \quad=$ Término de error
El coeficiente de determinación del modelo definitivo es 0.291 , indicando que $29 \%$ de la variabilidad en el índice de sitio es explicada por estas dos variables predictoras. El coeficiente de correlación en el modelo final es 0.54. Como lo indica el coeficiente Beta, el porcentaje de arena (0.410) tiene más influencia en el índice de sitio que el contenido de materia orgánica $(0.383)$

La figura 3 muestra el comportamiento de los residuos en el modelo y la variables independientes \%ARENA y MO. No se puede distinguir aumento de la variabilidad de los residuos, más bien su dispersión es obviamente homogénea, cumpliéndose así el postulado de regresión de homogeneidad.

\section{DISCUSIÓN Y CONCLUSIONES}

La modelización de la relación entre índice de sitio y propiedades del sitio se basa en la presunción de que los efectos de diferentes factores de crecimiento trabajan juntos de manera aditiva. El modelo propuesto se basa en los procesos biológicos implicados en el desarrollo de la altura dominante de un rodal boscoso de $P$. occidentalis Sw. Incluyó muchas variables, algunas de las cuales estuvieron fuertemente correlacionadas, lo cual dificulta la interpretación de los resultados.

Los datos utilizados están sujetos a varios errores. Se han realizado algunas investigaciones preliminares sobre los errores en los datos de índice de sitio, las propiedades del suelo y las topográficas. Los errores en la estimación de índice de sitio incluyen:

- Errores de predicción

- Errores de medición de la altura (Eriksson, 1970)

- Errores de estimación de la edad

En el presente estudio, las medidas de ajuste obtenidas por análisis de regresión indican una precisión insuficiente, pero los errores aleatorios no presentan sesgo en el análisis.

No se encontraron diferencias estadísticas significativas $(\alpha=0.05)$ para el índice de sitio obtenido mediante el modelo de Schumacher (1939) en las tres regiones de estudio. Para mejorar la predicción se ajustaron modelos separados con el índice de sitio como variable dependiente y 


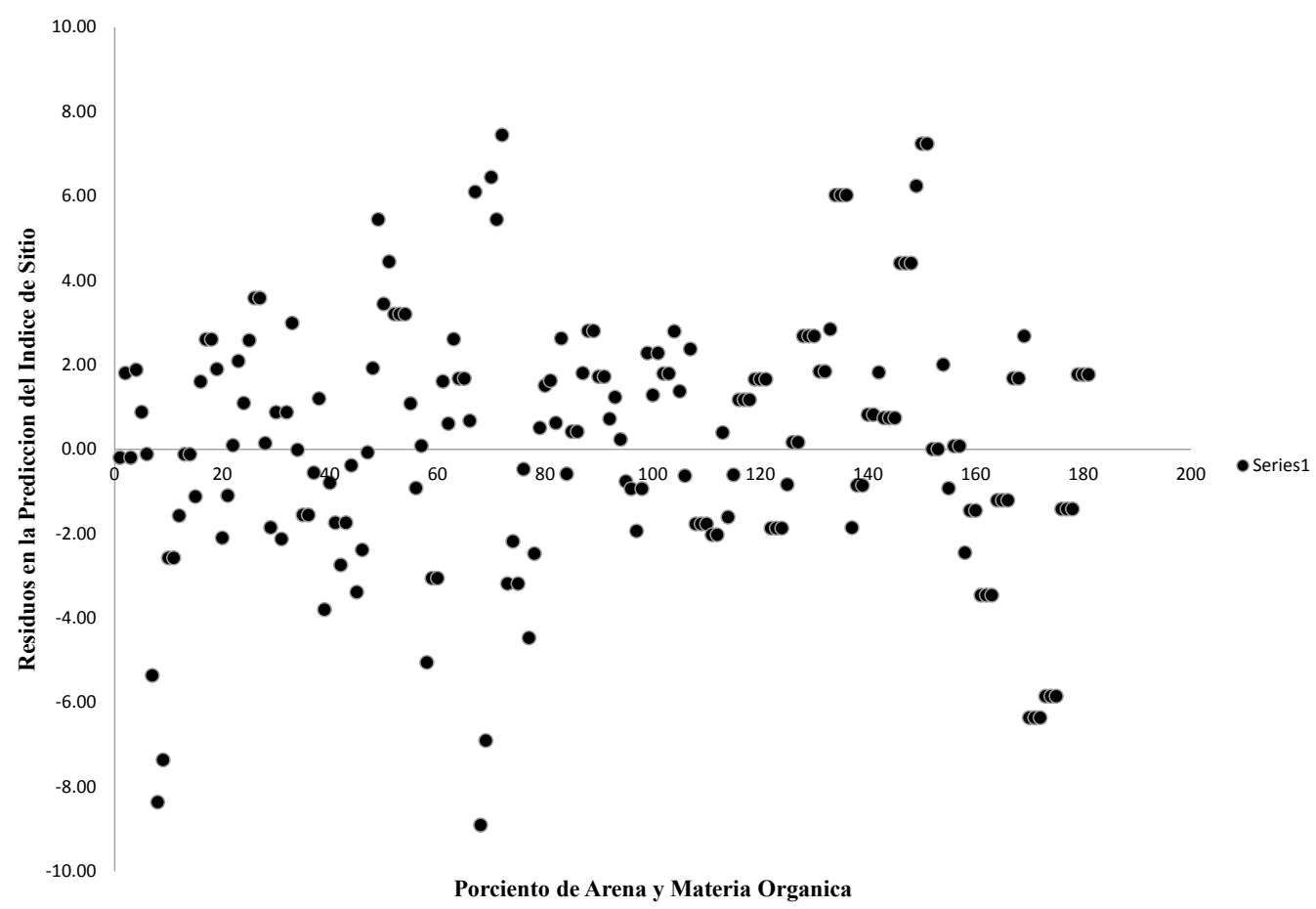

Figura 3. Gráfico parcial de residuos entre las variables Porciento de Arena (\%ARENA), contenido de materia orgánica (MO) y la variable dependiente Indice de Sitio, según el modelo (10).

variables topográficas, edáficas y de posición geográfica como predictivas.

Las variables geográficas elevación y azimut resultaron estadísticamente significativas al aplicar el procedimiento paso a paso, con iteraciones de entrada y remoción a nivel $\alpha$ de 0.01 y 0.05 respectivamente, para las pruebas F. Asimismo, las variables porciento de arena y contenido de materia orgánica resultaron estadísticamente significativas en la modelación parcial.

Los coeficientes de determinación de los modelos parciales resultaron muy bajos, $\mathrm{R}^{2}=0.14$ para el modelo [7] y los modelos [8] y [9], corridos con variables edáficas, $\mathrm{R}^{2}$ $=0.11$ y R ${ }^{2}=0.12$, respectivamente. Sin embargo, esas funciones son las que resultan con la menor suma de cuadrados.

El modelo final [10] resultó el mejor para predecir el valor de índice de sitio, utilizando el algoritmo de regresión paso a paso, la significancia estadística y $\mathrm{R}^{2}$. Este modelo puede ser utilizado sin la presencia de árboles para determinar el índice de sitio correspondiente en la zona de interés.

\section{RECONOCIMIENTOS}

Se agradece el apoyo recibido del Programa de Fondos Internos para Investigación de la Vicerrectoría de Investigación e Innovación de la Pontificia Universidad Católica Madre y Maestra. Se agradece el apoyo del Plan Sierra Inc. en la persona de su Director Ejecutivo el Ing. Luis Eduardo Peralta. Se agradece también al forestal Elvis Lizardo de la Escuela Ambiental del Ministerio de Recursos Naturales y Ambientales de la Republica Dominicana, por su valiosa colaboración. A los asistentes Juan Gilberto Torres, Eduardo Gómez y José Reyes, los pilares en la fase de campo, gratitud eterna.

\section{REFERENCIAS}

Andenmatten, E. y F. Letourneau. 2000. Curvas de índice de sitio y crecimiento en altura, para Pino Oregón (Pseudotsuga menziesii (Mirb)Franco), de aplicación en la región Andina. Revista Chapingo. Serie ciencias forestales y del ambiente 11(001):39-44. 
Avery, T.E. y H.E. Burkhart. 2002. Forest Measurements. McGraw Hill. Nueva York. 456p.

Bueno, S.W. 2009. Understanding growth and yield of Pinus occidentalis, Sw. in La Sierra, Dominican Republic. Disertación de grado de doctorado. State University of New York. College of Environmental Science and Forestry. Syracusa. Nueva York. 256 p.

Burgt, X.v.d. 1993. Determinación de la edad del Pinus occidentalis en la República Dominicana con la ayuda de sus anillos de crecimiento. Países Bajos. 127p.

Carmean, W.H. 1972. Site index curves for upland oaks in the Central States. Forest Science 18(2):109-120.

Clutter, J.L., J.C. Fortson, L.V. Pienaar, G.H. Brister y R.L. Bailey. 1983. Timber management: a quantitative approach. Krieger Publishing Company. Malabar, Florida. 233p.

Curtis, R.O., D.J. DeMars y F.R. Herman. 1973. Which dependent variable in site index-height-age regressions. Forest Science 52(2):173-181.

Dobler, G. 1999. Manejo y tablas de rendimiento de Pinus occidentalis. Servicio Alemán de Cooperación Técnica (DED). San José de las Matas, Republica Dominicana. 154p.

Dobler, G. 2000. Aspectos básicos de la silvicultura. San José de las Matas. $110 \mathrm{p}$.

Dobler, G., L.E. Peralta, R.T. Debord y J.G. Torres. 1999. Investigación y manejo de especies maderables de uso común en la sierra: una guía técnica. Plan Sierra. Servicio de Alemán de Cooperación Social-Técnica ded. 269p.

Elfving, B. y A. Kiviste. 2007. Construction of site index equations for Pinus sylvestris L. using permanent plot data in Sweden. Revista Bosque (Valdivia) 28(2):142-151.

Eriksson, H. 1970. On measuring errors in tree height determination mith different altimeters Inst for skogsproduktion, skogshogskolan. Rapporter och uppsatser $19 \mathrm{p}$. Forestalia Suecica 19(1):129-141.

Essea, C.R., C.O. Navarroa y J.C. Pinaresa. 2007. Curvas de índice de sitio para Nothofagus dombeyi en la zona preandina, provincia de Cautín, IX Región, Chile. Forest Science 52(2):198-208.

Farjon, A., J.A. Perez de la Rosa y B.T. Styles. 1997. A field guide to the pines of Mexico and Central America. The Royal Botanic Gardens. Kew. 78p.
Goelz, J.C.G. y T.E. Burk. 1992. Development of a well-behaved site index equation: jack pine in north central Ontario. Canadian Journal of Forest Research 22:776-784.

Holdridge, L. 1987. Ecología basada en zonas de vida. Instituto Interamericano de Cooperación para la Agricultura. San José, Costa Rica. 304 p.

Husch, B., T.W. Beers, J.A. Kershaw. 2003. Forest Mensuration. John Wiley \& Sons. Hoboken, Nueva Jersey. 433 p.

López-Hernández, E.N. 2010. Índice de sitio para Pinus patula Schl. et Cham., en Santiago Comaltepec, Ixtlán; Oaxaca. Forest Science 52(2):187-197.

Mathiasen R. L., W. K. Olsen y C. B. Edminster. 2006. Site index curves for White Fir in the Southwestern United States developed using a guide curve method. Forest Ecology and Management 98:125-134.

Monserud, R.A. 1984. Height growth and site index curves for Inland Douglas-fir Based on stem analysis data and forest habitat type. Forest Science 52(3):262-272.

Nigh, G. 1995. The geometric mean regression line: a method for developing site index conversion equations for species in mixed stands. Forest Science 41(1):84-98.

Nord-Larsen, T. 2006. Developing dynamic site index curves for European Beech (Fagus sylvatica L.) in Denmark. Forest Ecology and Management 145:151-161.

Nyland, R.D. 2002. Silviculture concepts and applications. 2a ed. Mc Graw-Hill. Nueva York. 682p.

SAS Institute Inc. 1990. SAS/STAT User's Guide. SAS Institute Inc. Cary, Carolina del Norte. 213 p.

Schumacher, F.X. 1939. A new growth curve and its applications to timber-yield studies. Journal of Forestry 37:819-820.

West, P.W. 2003. Tree and forest measurements. Springer. Dordrecht, Heidelberg, Londres, Nueva York. 167p.

Zhang, L., J.A. Moore y J.D. Newberry. 1993. Estimating asymptotic attributes of forest stands based on bio-mathematical rationales. Ecological Applications 3(4):743-748.

Manuscrito recibido el 30 de abril de 2014.

Aceptado el 15 de junio de 2015.

Este documento se debe citar como:

Bueno-López, S.W., J.G Torres H. y M. García. 2015. Factores edáficos-fisiográficos y calidad de sitio del Pinus occidentalis Sw. Madera y Bosques 21(3):83-93. 